\title{
Identificando as Atividades dos Grupos do Programa de Educação Tutorial na Área de Computação no Apoio à Inclusão e Alfabetização Digital
}

\author{
Emily Costa ${ }^{1}$, Antônio Pinto ${ }^{1}$, José Ribamar ${ }^{1}$, Alana Araújo ${ }^{1}$, Ednara Pereira ${ }^{1}$, \\ Geraldo Braz Júnior ${ }^{1,2}$, Rodrigo Santos ${ }^{3}$, Luis Rivero ${ }^{1,2}$ \\ ${ }^{1}$ Programa de Educação Tutorial de Ciência da Computação (PETComp) - \\ Universidade Federal do Maranhão (UFMA) \\ Av. dos Portugueses, 1966 - Vila Bacanga, São Luís - MA - Brasil \\ ${ }^{2}$ Programa de Pós-Graduação em Ciência da Computação (PPGCC) - Universidade \\ Federal do Maranhão (UFMA) \\ Av. dos Portugueses, 1966 - Vila Bacanga, São Luís - MA - Brasil \\ ${ }^{3}$ Programa de Pós-Graduação em Informática (PPGI) - Universidade Federal do Estado \\ do Rio de Janeiro (UNIRIO) \\ Av. Pasteur, 458 - Urca, Rio de Janeiro - RJ - Brasil \\ petcomputacao.ufma@gmail.com, geraldo@nca.ufma.br, rpseuniriotec.br
luisrivero@nca.ufma.br
}

\begin{abstract}
The Tutorial Educational Program (PET) aims to encourage groups of undergraduate students through extracurricular activities in Teaching, Research and Social Aid. There are 37 PET groups listed in the Brazilian Computer Science website, which report several activities supporting the community (people outside the university, elderly, children, others). This paper presents an analysis of the activities published by these groups aiming at supporting digital inclusion and literacy. The information on success cases of such activities can be used as basis for PET groups and encourage partnerships to carry out the activities.
\end{abstract}

Resumo. O Programa de Educação Tutorial (PET) tem o objetivo de incentivar grupos de alunos de graduação a desenvolver atividades relacionadas com o Ensino, a Pesquisa e a Extensão. Existem 37 grupos PET listados no site da $S B C$ que realizam atividades voltadas para a comunidade (público externo à universidade, idosos, crianças, entre outros). Este artigo apresenta uma análise de quais das atividades divulgadas por estes grupos são voltadas para o apoio à inclusão e alfabetização digital. As informações sobre casos de sucesso destas atividades podem servir como base para outros grupos e incentivar parcerias para realizar atividades afins.

\section{Introdução}

Segundo o relatório do censo da educação superior do Ministério da Educação ${ }^{1}$, alunos iniciantes em um curso de graduação enfrentam uma grande dificuldade em se manter em

\footnotetext{
${ }^{1}$ http://download.inep.gov.br/educacao_superior/censo_superior/apresentacao/2016/apre sentacao_censo_educacao_superior.pdf
} 
VIII Congresso Brasileiro de Informática na Educação (CBIE 2019)

Anais do XXV Workshop de Informática na Escola (WIE 2019)

um curso devido ao nível acadêmico exigido e à falta de acompanhamento acadêmico dos alunos. Visando diminuir estas dificuldades, o Programa de Educação Tutorial (PET) foi criado para aprimorar os cursos de graduação, inserindo estudantes em projetos de educação tutorial, com o objetivo de aplicar seus conhecimentos e sua formação [Martins 2007]. O objetivo deste programa é apoiar grupos de alunos que demonstrem potencial, interesse e habilidades destacadas em cursos de graduação, através de atividades extracurriculares que desempenhem os eixos de Pesquisa, Ensino e Extensão [Alberton e Amaral 2013].

No site da Sociedade Brasileira de Computação $(\mathrm{SBC})^{2}$, é relatada a existência de um total de 37 grupos PET para a área de Computação. Cada grupo é responsável por submeter um planejamento contendo as atividades que serão desempenhadas ao longo do ano e, ao fim do ano, escrever um relatório detalhando quais foram cumpridas e os resultados alcançados nos eixos descritos acima [Moraes e Castro Júnior 2017]. Dentre as atividades de extensão realizadas pelos grupos PET da área de computação, existe uma preocupação por parte dos membros dos grupos na realização de atividades que visam a inclusão e a alfabetização digital [Lucena et al. 2018][Ferreira et al. 2015]. A inclusão digital visa o planejamento e a execução de ações com o objetivo de permitir a participação de todos na sociedade da informação [Rodrigues et al. 2011]. Já a alfabetização digital diz respeito ao papel e à função das mídias e de outros provedores de informação, como bibliotecas, arquivos e internet, na vida privada das pessoas e nas sociedades democráticas [de Souza Silva et al. 2016].

Apesar da existência de trabalhos que relatam as atividades dos grupos PET de computação [Lucena et al. 2018][Ferreira et al. 2015], os autores deste trabalho não encontraram indícios da existência de trabalhos com foco nas diferentes ações para inclusão e alfabetização digital realizadas pelos programas PET para a área computação. Nesse contexto, considerando o potencial de disseminar e agregar o trabalho realizado pelos diferentes grupos PET de computação para oferecer uma análise mais aprofundada que permita a colaboração na realização destas atividades, este artigo apresenta uma análise das atividades desempenhadas por todos os PET listados pela SBC, focando nas atividades voltadas para inclusão e alfabetização digital e criando um catálogo com base nos resultados desta análise.

Além desta seção introdutória, este artigo está dividido em mais quatro seções. A Seção 2 apresenta os trabalhos relacionados com esta pesquisa. A Seção 3 apresenta a metodologia de pesquisa e o catálogo de atividades identificadas, classificando-as. A Seção 4 apresenta um resumo das atividades voltadas para a inclusão e alfabetização digital, descrevendo sua metodologia de execução. Finalmente, a Seção 5 apresenta as conclusões e perspectivas futuras para este trabalho.

\section{Trabalhos Relacionados}

No Brasil, a política pública de inclusão digital evidencia temas como cidadania, inclusão social, desenvolvimento local e nacional, em três eixos fundamentais: garantia de acesso às Tecnologias Digitais de Informação e Comunicação (TDIC); incentivo à disponibilização de conteúdos de interesse público; e capacitação da população e dos profissionais que atuam no atendimento ao cidadão [Rodrigues e Batista, 2018]. Por sua

\footnotetext{
${ }^{2}$ http://www.sbc.org.br/educacao/grupos-pet
} 
VIII Congresso Brasileiro de Informática na Educação (CBIE 2019)

Anais do XXV Workshop de Informática na Escola (WIE 2019)

vez, a alfabetização digital promove os direitos individuais de comunicação e de expressão, bem como de busca, recebimento e transmissão de informações e ideias. Ela encoraja a avaliação das mídias e de outros provedores de informação com base naquilo que é produzido, nas mensagens transmitidas e nos públicos visados [Wilson et al. 2013].

Com relação às atividades realizadas em termos de inclusão e alfabetização digital, vários trabalhos relatando ações têm sido publicados. Souza Silva et al. [2016], apresentam uma metodologia de alfabetização digital aos docentes que atuam na educação básica, relatando as lições aprendidas de um curso de formação inicial e continuada no Município de Floresta/PE. Por sua vez, Alves et al. [2015] discutem ações de extensão universitária para inclusão de recursos digitais no ensino de matemática a partir da experiência de dois alunos no âmbito do PROEXT com o projeto InfoMat Inclusão Digital no Ensino de Matemática em Escolas Públicas do Litoral Norte da Paraíba, realizado no ano de 2014. Os autores indicam as contribuições sobre a realidade escolar e desafios de um processo de inclusão nesses espaços. Em outro trabalho, Almeida Silva e Prado [2018] desenvolveram e apresentaram os resultados da validação de um instrumento que avalia o nível de conhecimentos e habilidades relacionados à Alfabetização Computacional.

Com relação às ações específicas dos grupos PET de computação levando em consideração inclusão e alfabetização digital, o trabalho de Lucena et al. [2018] apresenta os resultados de 7 ações realizadas no PETCC da Universidade do Estado do Rio Grande do Norte, como: apoio ao ensino através de monitorias, publicação de revista própria, semana acadêmica de ciência da computação, treinamentos da olimpíada brasileira de informática, reaproveitamento de lixo tecnológico, maratona de programação e apoio ao ensino de ferramentas nas escolas. Além disso, Ferreira et al. [2015] descrevem os resultados da metodologia aplicada no grupo PETComp no estado do Maranhão, onde são descritas atividades como aprendizado de tecnologias através do desenvolvimento de sistemas computacionais, aplicação de conhecimentos em áreas como robótica, e participação na inclusão digital através de treinamento em sistemas computacionais a pessoas da terceira idade.

Para que os grupos PET de computação do Brasil possam trabalhar de forma colaborativa e aprender com os casos de sucesso de iniciativas em inclusão e alfabetização digital, é necessário identificar e disseminar as ações já realizadas com um maior nível de detalhamento. Nesse contexto, a seguir é apresentado o processo de identificação das ações no contexto de inclusão e alfabetização digital.

\section{Identificação das Atividades de Inclusão e Alfabetização Digital}

Nesta seção, é apresentado o procedimento de identificação das atividades realizadas pelos grupos PET na área de Computação. Além disso, são listadas as atividades relacionadas com inclusão e alfabetização digital e seus resultados alcançados.

\subsection{Procedimento de Coleta de Dados dos Grupos PET}

Para catalogar as atividades desempenhadas pelos grupos PET de computação, optou-se pela consulta dos grupos listados no site da Sociedade Brasileira de Computação e no 
VIII Congresso Brasileiro de Informática na Educação (CBIE 2019)

Anais do XXV Workshop de Informática na Escola (WIE 2019)

Portal dos Grupos PET no MEC $^{3}$. Nestes sites, estão disponibilizadas informações de um total de 37 grupos PET na área de computação, contendo informações como: nome, instituição de ensino superior e portal oficial; divididos por região e listados em ordem alfabética. Os autores deste trabalho entraram na página oficial de cada grupo até março de 2019 para encontrar a descrição das atividades desempenhadas pelos grupos PET listados. No entanto, algumas das páginas oficiais dos grupos PET em computação estão fora de serviço ou desatualizadas. Consequentemente, foi registrado o problema de acesso à informação para estes grupos. Posteriormente, para cada grupo PET de computação que tivesse suas informações atualizadas (considerando como mínimo o ano de 2018), foram coletadas informações sobre suas atividades, indicando: nome da atividade, metodologia de execução e eixos da tríade (Ensino, Pesquisa e Extensão) aos que a mesma pertence.

Após este processo de identificação, considerando que diversos grupos PET utilizam nomes diferentes para se referir à mesma atividade, foi realizada uma análise das atividades visando identificar quais eram similares com base na sua descrição. Esta análise foi verificada por todos os autores do trabalho, com o objetivo de diminuir o risco de classificação incorreta. Além disso, quaisquer divergências de opinião eram resolvidas após uma discussão por parte do grupo e chegando a um consenso. Após a identificação das atividades e respectiva catalogação, as descrições foram agregadas, de forma a facilitar a replicação por parte de outras equipes de grupos PET. Estas informações foram concentradas em uma tabela para que os grupos PET com atividades similares pudessem verificar a possibilidade de realização de atividades comuns de forma colaborativa, além de permitir que grupos PET incorporem novas atividades e/ou inovem quanto às atividades realizadas em outros grupos. Na subseção a seguir, é apresentada a tabela e um resumo dos principais resultados identificados com relação às atividades voltadas para inclusão e alfabetização digital.

\subsection{Resultados}

Após uma consulta nos 37 grupos PET listados no site da SBC, 14 grupos tinham o site em manutenção ou indisponível e 6 grupos tinham seu site operacional, porém desatualizado. Sendo assim, foram consideradas as atividades de 17 grupos para análise, pois só estes tinham seus portais funcionando com informações atualizadas até 2018. Destes grupos, apenas 5 tinham suas informações atualizadas até o ano de 2019.

Ao todo, foi possível identificar um total de 18 atividades diferentes exercidas pelos 17 grupos PET de computação analisados. A Tabela 1 apresenta o catálogo de atividades ordenadas por frequência de ocorrência, indicando os grupos PET que as exercem e em quais universidades. As atividades foram agrupadas de maneira a sumarizar seu conteúdo, contando com uma breve descrição do que foi desempenhado. Por exemplo, a atividade "Semana do Fera", desempenhada pelo PET de Computação da UFCG (Universidade Federal de Campina Grande) foi catalogada como "Recepção de Calouros", assim como os outros 5 grupos PET também executaram trabalhos semelhantes, agrupados no mesmo título, como descrito na metodologia. Desta forma, ao longo do levantamento, foi possível relacionar atividades que ocorrem de maneira semelhante entre estes grupos, para assim poder delimitar quais são as possíveis formas

\footnotetext{
${ }^{3}$ http://portal.mec.gov.br/index.php?option $=$ com_docman\&view $=$ download\&alias $=5643$ -grupos-pet-2010\&category_slug=junho-2010-pdf\&Itemid=30192
} 
VIII Congresso Brasileiro de Informática na Educação (CBIE 2019)

Anais do XXV Workshop de Informática na Escola (WIE 2019)

de cooperação. Finalmente, as atividades foram ordenadas de acordo com a frequência em que ocorreram nos grupos PET, de modo a identificar quais atividades foram mais comuns entre os grupos PET.

Dentre as atividades identificadas, as atividades destacadas na Tabela 1: AT01 Oficinas e minicursos, AT02 - Eventos regionais, AT03 - Informática nas escolas, AT 11 - Iniciação científica, AT 13 - Atividades com idosos, e AT15 - Criação de Revista visam apoiar a inclusão e alfabetização digital de diversos públicos como crianças e adolescentes, idosos, pessoas com necessidades especiais, comunidade acadêmica e público em geral. Percebe-se que dos 17 grupos PET analisados, 16 realizam pelo menos uma das atividades relacionadas. Além disso, as atividades AT03 e AT13 que são mais ligadas à inclusão e alfabetização digital de crianças e idosos são realizadas em 10 dos 17 grupos analisados, indicando interesse por parte da comunidade PETiana neste tipo de ações. A seguir, as atividades relacionadas com inclusão e alfabetização digital e sua metodologia de execução serão descritas.

Tabela 1. Catálogo das Atividades dos Grupos PET de Computação,

\begin{tabular}{|c|c|c|c|c|c|c|c|c|c|c|c|c|c|c|c|c|c|c|}
\hline \multirow{2}{*}{$\begin{array}{l}\text { Universidade } \\
\text { / Curso }\end{array}$} & \multicolumn{18}{|c|}{ Atividade } \\
\hline & 1 & 2 & 3 & 4 & 5 & 6 & 7 & 8 & 9 & 10 & 11 & 12 & 13 & 14 & 15 & 16 & 17 & 18 \\
\hline $\begin{array}{c}\underline{\text { UFMA - }} \\
\underline{\text { Ciência da }} \\
\underline{\text { Computação }}\end{array}$ & $X$ & $\mathrm{X}$ & $X$ & $X$ & $\mathrm{X}$ & $X$ & & $X$ & & & & & & & & & & \\
\hline $\begin{array}{l}\frac{\text { UFC }- \text { Ciência }}{\underline{\text { da }}} \\
\underline{\text { Computação }}\end{array}$ & $\mathrm{X}$ & $X$ & $\mathrm{X}$ & & & $X$ & $X$ & & $X$ & & & $X$ & & & & & $\mathrm{X}$ & \\
\hline $\begin{array}{c}\text { UFRN - } \\
\underline{\text { Ciência da }} \\
\underline{\text { Computação }}\end{array}$ & $X$ & $X$ & & & $X$ & $X$ & $X$ & & & & & & & & & & & \\
\hline $\begin{array}{c}\text { UERN - } \\
\text { Ciência da } \\
\text { Computação }\end{array}$ & & $X$ & $X$ & & & & & & & $X$ & & & & $\mathrm{X}$ & & & & \\
\hline $\begin{array}{c}\underline{\text { UFCG - }} \\
\underline{\text { Ciência da }} \\
\underline{\text { Computação }}\end{array}$ & $X$ & $X$ & $X$ & $X$ & $X$ & $X$ & & & & & & & & & & $X$ & & \\
\hline$\frac{\underline{\text { UFPB - }}}{\text { Ciência da }}$ & $X$ & & $X$ & $X$ & $X$ & & & & $X$ & $X$ & & & $X$ & & & & & \\
\hline $\begin{array}{l}\underline{\text { UESC - }} \\
\underline{\text { Controle e }} \\
\text { automação }\end{array}$ & $X$ & $X$ & $X$ & & & & & & $X$ & & & & $X$ & & & & & \\
\hline $\begin{array}{l}\underline{\text { UFG - }} \\
\text { Engenharias }\end{array}$ & & $X$ & & & & & & $X$ & & & & & & & & & & \\
\hline $\begin{array}{c}\text { UFMS - } \\
\text { Fronteira } \\
\end{array}$ & $X$ & & & & & & & & & & & & & & & & & \\
\hline$\frac{\text { UFU - }}{\text { Fronteira }}$ & $X$ & $X$ & & & & & $X$ & & & & & & & & & & & \\
\hline$\frac{\text { USP - }}{\text { Sistemas de }}$ & $X$ & & & & & $\mathrm{X}$ & $\mathrm{X}$ & $X$ & & & & & & & & & & \\
\hline$\frac{\text { Unioeste - }}{\text { Informática }}$ & $X$ & & $X$ & $X$ & & & & & & & & & & $\mathrm{X}$ & & & & \\
\hline
\end{tabular}


VIII Congresso Brasileiro de Informática na Educação (CBIE 2019)

Anais do XXV Workshop de Informática na Escola (WIE 2019)

\begin{tabular}{|c|c|c|c|c|c|c|c|c|c|c|c|c|c|c|c|c|c|c|}
\hline \multirow{2}{*}{$\begin{array}{l}\text { Universidade } \\
\text { / Curso }\end{array}$} & \multicolumn{18}{|c|}{ Atividade } \\
\hline & 1 & 2 & 3 & 4 & 5 & 6 & 7 & 8 & 9 & 10 & 11 & 12 & 13 & 14 & 15 & 16 & 17 & 18 \\
\hline 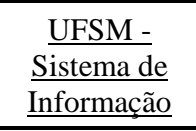 & $X$ & & $X$ & $X$ & $X$ & $X$ & $X$ & $X$ & & & $\mathrm{X}$ & & & & $X$ & $\mathrm{X}$ & & \\
\hline 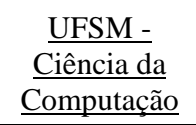 & & & & $\mathrm{X}$ & $X$ & & & & $\mathrm{X}$ & & $\mathrm{X}$ & & $\mathrm{X}$ & & & & & $\mathrm{X}$ \\
\hline$\frac{\frac{\text { UTFPR - }}{\text { Engenharia da }}}{\underline{\text { Computação }}}$ & & & & & $X$ & & & $X$ & $\mathrm{X}$ & & & $\mathrm{X}$ & & & & & & \\
\hline$\frac{\text { UFSC - }}{\underline{\text { Ciência da }}} \underline{\text { Computação }}$ & $X$ & $X$ & $\mathrm{X}$ & $X$ & $X$ & & $\mathrm{X}$ & & & $\mathrm{X}$ & & $\mathrm{X}$ & & & $X$ & & & \\
\hline$\frac{\underline{\text { UFPel - }}}{\underline{\text { Ciência da }}}$ & $X$ & & & $\mathrm{X}$ & & $X$ & $\mathrm{X}$ & $X$ & & $\mathrm{X}$ & $\mathrm{X}$ & & & & & & & \\
\hline $\begin{array}{c}\text { Total de } \\
\text { Ocorrências }\end{array}$ & 13 & 9 & 9 & 8 & 8 & 7 & 7 & 6 & 5 & 4 & 3 & 3 & 3 & 2 & 2 & 2 & 1 & 1 \\
\hline
\end{tabular}

Legenda: AT 01 - Oficinas e minicursos, AT 02 - Eventos regionais, AT 03 - Informática nas escolas, AT 04 - Desenvolvimento de software, AT 05 - Monitorias, AT 06 - Recepção dos calouros, AT 07 - Competições e Preparatório para a Maratonas, AT 08 Confraternizações e debates, AT 09 - Sistemas embarcados, AT 10 - Biblioteca, AT 11 - Iniciação científica, AT 12 - Grupo de estudos sobre Sistemas Operacionais, AT 13 - Atividades com idosos, AT 14 - Reciclagem de lixo eletrônico, AT 15 - Criação de Revista, AT 16 - Incentivo ao empreendedorismo, AT 17 - Curso Preparatório para obter certificação e AT 18 - Preparatório póscomp.

\section{Processo de Aplicação das Atividades Identificadas}

Para entender melhor como implementar as atividades relacionadas com inclusão e alfabetização digital, esta seção apresenta uma breve descrição da metodologia de cada atividade. Adicionalmente, são descritos os objetivos da atividade, o público alvo que atende e os eixos em que a mesma se encaixa.

\subsection{AT 01 - Oficinas e Minicursos}

Um minicurso é uma proposta de curso de curto prazo (entre 3 e 6 horas), introduzindo técnicas e conhecimentos com aplicações fora da sala de aula. Durante o levantamento percebeu-se que 13 grupos PET ministram rotineiramente minicursos, tendo como público alvo: alunos de graduação e pós-graduação, ex-alunos e público externo à universidade. Esta atividade, por ser aplicada dentro do eixo de ensino, abrange assuntos gerais e específicos da computação e os aplicam das formas mais variadas.

Nesta atividade, por exemplo, o PET de Computação da Universidade Federal do Maranhão - UFMA desenvolve a atividade "Grupo de Saberes" cujos temas abordam as tecnologias e conhecimentos trabalhados em outras atividades do grupo, como a de desenvolvimento de software, uso de softwares variados, ou conteúdo aprendido durante as iniciações científicas dos alunos.

Seguindo esta linha de pensamento, o PET da Universidade Federal de Campina Grande realiza palestras e minicursos abordando variados temas dentro da computação. É importante ressaltar que a realização dessa atividade pode ser benéfica tanto para o ministrante quanto para ouvinte, colocando em perspectiva que a maior parte dos ministrantes exercitam a prática didática e os próprios conhecimentos que estão sendo passados durante o curso. 
VIII Congresso Brasileiro de Informática na Educação (CBIE 2019)

Anais do XXV Workshop de Informática na Escola (WIE 2019)

\subsection{AT 02 - Eventos Regionais}

Esta atividade contempla os grupos PET que trabalham apoiando e/ou realizando eventos regionais ou nacionais. Essas atividades contemplam, geralmente, todos os eixos de ensino, pesquisa e extensão, encaixando perfeitamente com a tríade seguida pelos grupos PET. Dentro destes eventos os principais acontecimentos envolvem palestras, mostra de trabalhos, demonstrações de tecnologias atuais, e trocas de experiência entre alunos, professores e profissionais atuando fora da universidade.

Um evento científico regional tem grande relevância no contexto local, pois oportuniza pesquisadores a expor seus trabalhos e abrir discussões sobre o tema através da comunicação oral e exposição de artigos científicos. Através destas modalidades básicas os participantes têm a oportunidade de divulgar seu trabalho, receber apreciações gerais, sugestões, críticas, além de possibilitar a ampliação do rol de interlocutores.

Entre os grupos pesquisados, 9 deles se envolvem com pelo menos um evento regional. Por exemplo, no PET Sistemas de Informação da universidade UFSC (Universidade Federal de Santa Catarina), existe o evento SECCOM (Semana Acadêmica de Ciências da Computação e Sistemas de Informação), voltado para os cursos de ciência da computação e sistemas de informação, onde são realizados minicursos, palestras e mostras tecnológicas, agregando conhecimentos específicos e perspectiva no mercado de trabalho após a graduação, através de uma feira empresarial.

\subsection{AT 03 - Informática nas Escolas}

Barcelos et al. [2009] apontam que o ensino de algoritmos e lógica de programação nas instituições tem sido constantemente objeto de estudos, visando a melhoria do desempenho dos estudantes, pois este conteúdo é considerado de suma importância para a trajetória acadêmica promovendo o ensino-aprendizagem. Além disso, Barcelos et al. [2009] indicam a importância de incentivar alunos de ensino fundamental e médio no ensino de computação, e instigar o interesse pela área de tecnologia da informação para melhorar o desempenho dos estudantes. A atividade de informática nas escolas envolve os eixos de ensino e extensão.

Atualmente, apesar da tecnologia estar presente no cotidiano de alguns dos alunos, ainda existem alunos que não estão inseridos no âmbito da computação ou estudam em escolas que não possuem uma estrutura para incentivo ao aprendizado deste conhecimento. Tendo isso em vista, foi apurado que 9 grupos PET praticam alguma atividade que envolve levar assuntos de informática para turmas de ensino fundamental e médio.

O PET da Universidade Federal da Paraíba - UFPB, visando a divulgação dos cursos do Centro de Informática da UFPB e disseminação do conhecimento de programação, oferece cursos e seminários para alunos do ensino médio, tendo como principais temas a introdução à computação e apresentação de cursos como Engenharia da Computação, Matemática Computacional e Bacharelado em Ciência da Computação.

Seguindo esse pensamento, mas visando uma abordagem lúdica, o PET de controle e automação da Universidade Estadual de Santa Cruz promove a "Oficina de Robótica Educacional" que aborda o ensino-aprendizagem aplicando conceitos de coletividade, cooperação e motivação entre os grupos de alunos, através do 
VIII Congresso Brasileiro de Informática na Educação (CBIE 2019)

Anais do XXV Workshop de Informática na Escola (WIE 2019)

desenvolvimento de kits de robótica de baixo custo. Por meio dessas atividades, é possível introduzir o jovem na área de computação e levar educação de qualidade.

\subsection{AT 11 - Iniciação Científica}

Nesta atividade do eixo de pesquisa, os projetos de iniciação científica trazem ao discente uma perspectiva diferente diante de um curso de graduação, os quais, além do ambiente da sala de aula, permitem ao aluno passar por um processo de aprendizado voltado à uma linha de pesquisa de sua escolha e habituar-se a metodologias científicas para obter soluções para um determinado problema. Eventualmente a maioria dos grupos PET pesquisados submetem trabalhos com base em suas atividades, porém durante $\mathrm{o}$ levantamento de dados, foi constatado que apenas 3 grupos consideram as pesquisas de iniciação científica uma atividade constante do grupo, ou seja, os outros grupos PETs submetem trabalhos casualmente, mas não alocam tempo dedicado para pesquisas.

Entre os grupos PET, foi possível estabelecer três quais possuem linhas de pesquisa bem definidas. Entre eles, por exemplo, temos o PET de Computação da UFPel (Universidade Federal de Pelotas), que desenvolve pesquisas em sistemas digitais, sistemas embarcados, inteligência artificial e sistemas paralelos e distribuídos. Seguindo essa mesma linha, porém em áreas mais diversas, conseguimos encontrar o PETComp da UFMA que desenvolve pesquisa em robótica, interação humano-computador, processamento de linguagem natural, engenharia de software, entre outros. Nesse contexto, existem pesquisas sendo realizadas nestes grupos que visam a melhoria da qualidade de software para uso por distintos grupos de usuários, como usuários com deficiência física ou cognitiva, como é o caso do trabalho de Mendes et al. [2018] em que foi realizada uma pesquisa para a proposta de uma interface específica para usuários idosos do aplicativo Whatsapp.

\subsection{AT 13 - Atividades com Idosos}

A realização de atividades com pessoas da terceira idade se classifica dentro da tríade como um projeto de extensão, visto que ocorre a inclusão de parte da sociedade durante a execução do trabalho. Apesar de ser uma ação que contribui bastante dentro da sociedade, observamos que apenas 3 grupos PET apresentam atividades de inclusão de idosos, dentre eles se encontra o PET da Universidade Federal de Santa Maria - UFSM que tem um projeto chamado "AcampaVida", que busca abrir oportunidades de convivência dentro da universidade, com atividades físicas, culturais e de lazer. Por sua vez, o PET da Universidade Federal da Paraíba realiza a atividade "Informática Básica para idosos", em que eles vão aprender os conceitos mais básicos sobre a computação, como por exemplo, entender como funciona um computador e suas funcionalidades para o dia-a-dia.

\subsection{AT 15 - Revista}

A cobertura e divulgação das atividades dos grupos PETs é muito importante para a veiculação de informações e/ou inscrições de atividades, além de contribuir na difusão dos trabalhos e das pesquisas realizadas dentro dos grupos. As atividades de divulgação enquadram-se, principalmente, no eixo de extensão, uma vez que são abertas à comunidade fora do âmbito acadêmico, disponibilizando ao público externo $\mathrm{o}$ conhecimento adquirido através do ensino e da pesquisa desenvolvidos pelos discentes. 
VIII Congresso Brasileiro de Informática na Educação (CBIE 2019)

Anais do XXV Workshop de Informática na Escola (WIE 2019)

Como forma de divulgação de informações alguns grupos utilizam revistas criadas por eles. Por exemplo, o projeto de "DivulgaPET" oferecido pelo PET-SI da Universidade Federal de Santa Maria - UFSM consiste na realização de encontros com pesquisadores que atuam em cursos na área de computação, dessa forma possibilitando a divulgação de pesquisas dos petianos e dos outros pesquisadores da área Sistemas de informação da Universidade. Outro exemplo da atividade é a "Revista Abstração" desenvolvida pelo PET Computação da Universidade Federal de Santa Catarina - UFSC que tem o objetivo de incentivar os discentes a elaborar artigos científicos para serem publicados no periódico distribuído em PDF e veiculado no site do mesmo.

\section{Conclusões}

Este trabalho apresentou um mapeamento das diversas atividades executadas pelos grupos PET listados pela SBC através de dados públicos extraídos de suas respectivas páginas oficiais, focando especificamente nas atividades relacionadas com a inclusão e alfabetização digital. Foi realizada uma análise no que diz respeito aos objetivos, metodologia, e quando disponibilizado, seus resultados. Diante de uma grande quantidade de dados, as atividades semelhantes foram agrupadas em categorias para melhor analisálas.

O principal enfoque dos grupos PET está no ensino, seguido pela extensão e finalizando com a pesquisa, considerando o enfoque das atividades identificadas. As 5 atividades mais comuns aos grupos PET (com ocorrência de até 8 grupos) foram: Realização de Oficinas e minicursos, Realização de Eventos, Apoio no ensino de Informática nas Escolas, Práticas em Projetos de Desenvolvimento de software e Monitorias. Além disso, dos 17 grupos PET analisados, 16 realizam pelo menos uma das atividades relacionadas à inclusão e alfabetização digital: AT 01 - Oficinas e minicursos, AT 02 - Eventos regionais, AT 03 - Informática nas escolas, AT 11 - Iniciação científica, AT 13 - Atividades com idosos, e AT 15 - Criação de Revista. A descrição das atividades pode servir de base para que grupos PET possam realizar parcerias e/ou iniciar a execução das mesmas. Por exemplo, quanto aos minicursos, os mesmos podem ser gravados e disponibilizados aos alunos que participaram do mesmo e/ou abertos ao público externo, ou pode ser preparada uma sala online para que haja uma troca de experiências e discussões. Além disso, os grupos PET que queiram inovar quanto às suas atividades podem utilizar estas atividades como base e aprender de experiências prévias, incorporando estas atividades na sua metodologia.

Como trabalhos futuros, está em perspectiva contatar os grupos PET referenciados. Dessa maneira, espera-se comunicar os pontos a serem melhorados em cada uma das atividades, através de um processo de colaboração. Além disso, pretendese utilizar os resultados desta pesquisa para propor uma metodologia anual para os grupos PET que permita manter equilíbrio na tríade do ensino, pesquisa e extensão, visando também a realização de atividades de apoio à inclusão e à alfabetização digital. Adicionalmente, pretende-se fazer uma avaliação do impacto das atividades realizadas, entrando em contato com os grupos PET executores e identificando oportunidades de melhoria nas mesmas. Espera-se com isso, apoiar os grupos PET na área de Computação no planejamento de suas atividades, adaptando-as à realidade do seu contexto, e melhorando o seu desempenho no apoio à sociedade. 
VIII Congresso Brasileiro de Informática na Educação (CBIE 2019)

Anais do XXV Workshop de Informática na Escola (WIE 2019)

\section{Referências}

Alberton, B. A. V. and Amaral, M. A. (2013). Oficinas de Robótica para Alunos do Ensino Médio: introduzindo a computação para futuros ingressantes. In Anais dos Workshops do Congresso Brasileiro de Informática na Educação (Vol. 2, No. 1).

Alves, E., Assis, C. and Martins, F. S. (2015, October). Inclusão Digital e o Ensino de Matemática em Escolas Públicas: Vivências no Programa Infomat/Proext. In Anais do Workshop de Informática na Escola (Vol. 21, No. 1, p. 340).

Barcelos, R., Tarouco, L. and Bercht, M. (2009). O uso de mobile learning no ensino de algoritmos. RENOTE, 7(3), 327-337.

de Almeida Silva, W. R. and Prado, E. (2018, October). Criação de Instrumento para Avaliação da Alfabetização Computacional. In Anais do Workshop de Informática na Escola (Vol. 24, No. 1, p. 670).

de Lucena, L. B., de Andrade, G. L. C., Soares, E. D. F. A., de Oliveira, Á. G. G., Batista, L. M. D. S. D., Gomes, D. A. and de Lima, R. W. (2018,). As Ações do Pet no Desenvolvimento do Curso de Ciência da Computação. In $26^{\circ}$ Workshop sobre Educação em Computação (WEI 2018), SBC.

de Souza Silva, J., Silva, E. and de Albuquerque, C. H. (2016, November). Alfabetização Digital para Professores da Educação Básica: Um Relato de Experiência. In Anais do Workshop de Informática na Escola (Vol. 22, No. 1, p. 855).

Ferreira J., Ribeiro, J., Freitas, A., Barros, G., Santos, I., Santos, H., Chaves, M., Albuquerque, A., Silva, B., Rezende, L., Bezerra, V., Moraes, A., Oliveira, A. (2015) Educação Tutorial em Ciência da Computação: uma proposta de sistematização $23^{\circ}$ WEI - Workshop sobre Educação em Computação.

Martins, I. L. (2007). Educação Tutorial no ensino presencial: uma análise sobre o PET. PET-Programa de Educação Tutorial: estratégia para o desenvolvimento da graduação. Brasília: Ministério da Educação.

Mendes, J., Santos Junior, J., Trindade, W., Fernandes, A., Rivero, L., Braz Junior, G. (2018). Reprojeto da Interface do WhatsApp Considerando o Usuário da Terceira Idade. In: VI Jornada de Informática do Maranhão, 2018, São Luís. ANAIS JIM UFMA - Jornada de Informática do Maranhão, 2018.

Moraes, L. M. P. and de Castro Júnior, A. A. (2017). PET Fronteira: Concepção, Ações e Objetivos. Revista ComInG-Communications and Innovations Gazette, 2(1), 35-45.

Rodrigues, N. and Batista, M. (2018, October). Ações e Políticas Públicas de Inclusão Digital: do global ao local, através de conceitos e processos educacionais. In Anais do Workshop de Informática na Escola (Vol. 24, No. 1, p. 624).

Rodrigues, N.N. et al. (2011) Tecnologias de Informação e Comunicação Aplicadas à Terceira Idade. Workshop de Informática na Escola, Aracajú.

Wilson, C. Grizzle, A. Tuazon, R. Akyempong, K. Cheung, C. (2013) "Alfabetização midiática e informacional: currículo para formação de professores", Brasília: UNESCO, UFTM 